\title{
Partial-Order Reduction for General State Exploring Algorithms
}

\author{
Dragan Bošnački ${ }^{1}$, Stefan Leue $^{2}$, and Alberto Lluch Lafuente ${ }^{3}$ \\ ${ }^{1}$ Eindhoven University of Technology, \\ Den Dolech 2, P.O. Box 513, \\ 5612 MB Eindhoven, The Netherlands \\ ${ }^{2}$ Department of Computer and Information Science, \\ University of Konstanz, \\ D-78457 Konstanz, Germany \\ ${ }^{3}$ Via del Giardino A 58, \\ I 50053 Empoli (FI), Italy
}

\begin{abstract}
An important component of partial-order based reduction algorithms is the condition that prevents action ignoring, commonly known as the cycle proviso. In this paper we give a new version of this proviso that is applicable to a general search algorithm skeleton also known as the General State Expanding Algorithm (GSEA). GSEA maintains a set of open (visited but not expanded) states from which states are iteratively selected for exploration and moved to a closed set of states (visited and expanded). Depending on the open set data structure used, GSEA can be instantiated as depth-first, breadth-first, or a directed search algorithm. The proviso is characterized by reference to the open and closed set of states in GSEA. As a result the proviso can be computed in an efficient manner during the search based on local information. We implemented partial-order reduction for GSEA based on our proposed proviso in the tool HSF-SPIN, which is an extension of the model checker SPIN for directed model checking. We evaluate the state space reduction achieved by partial-order reduction according to the proviso that we propose by comparing it on a set of benchmark problems to other reduction approaches. We also compare the use of breadth-first search and $A^{*}$, two algorithms ensuring that counterexamples of minimal length will be found, together with the proviso that we propose.
\end{abstract}

\section{Introduction}

Partial-Order Reduction (POR) 4, 8, 22, 23, 25, 26] is one of the main techniques used to tackle the state explosion problem in model checking. An important component of partial-order based reduction algorithms is the condition that prevents action ignoring, commonly known as the cycle proviso. In this paper we give a new version of this proviso that is applicable to a general state search algorithm skeleton also known as the General State Exploring Algorithm (GSEA) which maintains a set of open (visited but not expanded) states from which states 
are iteratively selected for exploration and moved to a closed set of states (visited and expanded).

Unlike the full state space exploration, POR expands only a subset of the enabled actions in a given state, called the ample set. The actions outside the ample set are temporarily ignored. However, if one is not careful, an action could be permanently ignored along some cycle in the reduced state space. Consider a state $s$ that appears in both the full and the reduced state spaces. An action $a$ is (permanently) ignored if it is executed in $s$ in the full state space, but it is ignored along all execution sequences starting at $s$ in the reduced state space.

To prevent this, we require that the following condition (which we call open set proviso) is satisfied: at least one state $s$ which is directly reachable via an action from the ample set has not been visited before or it is in the set of open states. Otherwise the ample set consists of all enabled transitions. For simplicity, in the remainder of this introductory section we treat the newly generated unvisited states also as open states since they will eventually be entered in the open set.

The intuition behind the open set proviso is that the ignoring problem is postponed until state $s$ is expanded later. As the ignored actions are independent of the actions in the ample set, they stay enabled in the open state. Thus, they will be either selected in the ample set of $s$ and as such executed, or they will be delayed for another open state reachable from $s$. Under the assumption that the GSEA algorithm terminates one can show that this postponement will eventually stop. This is because the set of open states will eventually become empty.

Such a proviso is a generalization of the cycle proviso for partial-order reduction with breadth-first search (BFS) 2] implemented in the model checker SPIN. The BFS POR proviso in turn was inspired by the algorithm presented in [1] for the application of POR in symbolic state space exploration.

Being characterized by means of the open set of states in GSEA, the open set proviso can be computed in an efficient manner during the search based on local information, i.e., information about the currently expanded state and its successors. Further, depending on the data structure which is used to represent the open set, GSEA can be instantiated as a depth-first, a breadth-first, or a directed search algorithm. As it was shown in [5], the latter can significantly improve the error-detection capabilities of explicit state model checking.

We implemented partial-order reduction for GSEA based on our proposed proviso in the tool HSF-SPIN, which is an extension of the model checker SPIN for directed model checking. We evaluate the state space reduction achieved by partial-order reduction according to the proviso that we propose by comparing it on a set of benchmark problems to other reduction approaches.

With the development of a proviso that is applicable to BFS as well as $\mathrm{A}^{*}$, which is an optimal directed heuristic search algorithm if an admissible heuristics is used, we can experimentally address a further relevant issue. When checking safety properties both BFS and $\mathrm{A}^{*}$ are capable of returning counterexamples of minimal length if an erroneous state is found in the state space. The usage of BFS without partial order reduction is often impossible due to the memory 
needs of this algorithm. But this obstacle to its application is partially remedied by the availability of an efficient partial order reduction, which this paper (as well as some previous papers) offers. It will hence be interesting to see how both optimal algorithms perform when used to find errors with the proposed proviso.

Related Work. The POR algorithm of [1] is for symbolic state space exploration and as such it is based on BFS. Unlike the POR version of GSEA (and the open set proviso, as a part of it) which is presented in this paper, the algorithm proposed in [1] is not dealing with reopening of states. Further, the practical side of the theory in [1] hinges on the concept of history function which assigns to each state a set of states.

The states in the history can be seen as potentially "dangerous" because they can lead to a cycle. By requiring that at least one action leads outside the "dangerous" set, i.e., at least one successor state does not belong to the history, one ensures that at least one action from the ample set does not close a cycle. (Therefore, the temporarily ignored transitions can safely be postponed.) In order to be useful in practice, there should be a simple criterion to define such history sets. For example, in the context of explicit state model checking, assuming depth-first search (DFS) exploration, the history set of the currently expanded state $s$ consists of the states which are on the DFS stack. If at least one of the successors is not on the DFS stack we are sure that at least one transition from the ample set does not close a cycle.

To avoid cycles, the definition of history requires that for no two states $s, s^{\prime}$, $s$ belongs to the history of $s^{\prime}$ and, vice versa, $s^{\prime}$ is in the history of $s$. Because of the reopening of states that GSEA performs, a direct application of the history concept is not possible since the set of open states does not satisfy such a requirement. Our approach, however, results in an efficiently checkable condition which is still expressed in terms of the set of open (closed) states.

In [5] a simple proviso is proposed. It requires that at least one newly generated state is not one of the already visited states. As the set of open states is a subset of the visited states, the open set proviso is weaker than the visited proviso. As a result reductions which are refuted by the visited proviso are allowed by the open set proviso. Our experiments show that this leads to significantly better results than the ones presented in [5].

In another work 14, the authors exploit the fact that the concurrent systems we work with are defined by a parallel composition of sequential processes. This leads to the formulation of a static version of the cycle proviso. This variant of the proviso does not depend on the search status but on information regarding control flow cycles of component processes that is gathered at compile-time. This static proviso is in general much stronger than the previously discussed provisos. Nonetheless, as our experiments showed, in practice it tends to be less efficient than the open set proviso that we introduce in this paper.

Alternatives for the cycle proviso are presented in [17] and [16. Both references assume DFS exploration of the state space and are therefore not directly applicable to our setting. Shortly before the submission of this paper we were made aware of an adaptation for breadth-first search of the algorithm in [17 
described in 20]. The very short description of the POR algorithm in 20] does not provide sufficient detail to allow for a meaningful comparison with our approach. However, reconciling this approach with ours might be an interesting subject for future research.

Paper Outline. In Section 2 we review the foundations of labeled transition systems, partial-order reduction and directed model checking. Our approach towards an efficient partial-order reduction for general state space exploring algorithms is introduced in Section 3. We describe our experimental results in Section 4 and conclude in Section 5 .

\section{Preliminaries}

\subsection{Transition Systems}

Our approach mainly targets the verification of asynchronous systems where the global system is constructed as an asynchronous product of a set of local component processes. We assume an interleaving model of execution. To reason formally about such systems, we introduce the notion of a labeled transition system 1

Definition 1 (Labeled transition system). A labeled transition system (LTS), is a 6-tuple $(S, \hat{s}, A, \tau)$, where $S$ is a finite set of states, $\hat{s} \in S$ is the initial state, $A$ is a finite set of actions, and $\tau: S \times A \rightarrow S$ is a (partial) transition function.

Let $\mathcal{T}=(S, \hat{s}, A, \tau)$ be an LTS. An action $a \in A$ is said to be $\mathcal{T}$-enabled in state $s \in S$, denoted $s \stackrel{a}{\rightarrow} \mathcal{T}$ iff $\tau(s, a)$ is defined. The set of all actions $a \in A$ enabled in state $s \in S$ is denoted enabled $\mathcal{T}_{\mathcal{T}}(s)$; that is, for any $s \in S$, enabled $_{\mathcal{T}}(s)=\{a \in$ $A \mid s \stackrel{a}{\rightarrow} \mathcal{T}\}$. When the LTS is clear from the context we omit the $\mathcal{T}$ subscript. A state $s \in S$ is a deadlock state iff $\operatorname{enabled}(s)=\emptyset$.

The transition function $\tau$ of LTS $\mathcal{T}$ induces a set $T \subseteq S \times A \times S$ of transitions defined as $T=\left\{\left(s, a, s^{\prime}\right) \mid s, s^{\prime} \in S \wedge a \in A \wedge s^{\prime}=\tau(s, a)\right\}$. To improve readability, we write $s \stackrel{a}{\rightarrow} s^{\prime}$ for $\left(s, a, s^{\prime}\right) \in T$. We also say that $s^{\prime}$ is a successor of $s$.

The transition function $\tau$ implies that the LTSs are deterministic in the sense that in a given state $s$ an action $a$ cannot result in more than one state. However, this is not a restriction from a practical point of view, as we shall now argue. Note that in practice the labels of the transitions correspond to program statements (see [11, for instance). Consider first two statements which are the same but belong to two different processes. As an example, this is the case if we have two instances of the same statement that belong to different instances of the same concurrent process (proctype, in SPIN). If the statement does not change the program (location) counter, then the theoretical condition

\footnotetext{
${ }^{1}$ Labeled Transition Systems with state propositions, like the ones used in this paper, are sometimes named "Labeled Kripke structures" or "Doubly labeled transition systems".
} 
that $\tau(s, a)$ always results in the same state is trivially satisfied. Suppose that in a given (global) state $s$ the execution of the statement that corresponds to action $a$ changes the program (location) counter of the process to which it belongs. Then, since the program counters are part of the state vector, the execution of each statement results in a different global state. In case we have non-determinism within the same process, it does not make much sense to have statements with the same name within the same non-deterministic choice. For instance, consider the following code in Promela, the input language of Spin:

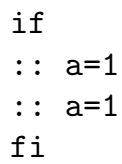

Depending on the implementation, in such a case each statement would either have a unique identifier or the statements would automatically be merged into one statement, such as this would be done in Spin. A similar argument can be made regarding non-determinism in other contexts, like process algebra. As an example consider non-observable actions obtained as a result of hiding. Translated into Promela they become skip actions that only affect the program counter. An analogous argument as above also applies to this case.

An execution sequence of an LTS $\mathcal{T}$ is a (finite) sequence of consecutive transitions in $T$. For any natural number $n \in \mathbb{N}$, states $s_{i} \in S$ and actions $a_{i} \in A$ with $i \in \mathbb{N}$ and $0 \leq i<n, s_{0} \stackrel{a_{0}}{\rightarrow} s_{1} \stackrel{a_{1}}{\rightarrow} \ldots s_{n-1} \stackrel{a_{n-1}}{\rightarrow} s_{n}$ is called an execution sequence of length $n$ of $\mathcal{T}$ iff $s_{i} \stackrel{a_{i}}{\rightarrow} s_{i+1}$ for all $i \in \mathbb{N}$ with $0 \leq i<n$. State $s_{n}$ is said to be reachable from state $s_{0}$. A state is said to be reachable in $\mathcal{T}$ iff it is reachable from $\hat{s}$.

\subsection{Partial-Order Reduction}

The basic idea of state space reduction is to restrict the part of the state space of a concurrent system that is explored during verification in such a way that all properties of interest are preserved. Partial-order reduction exploits the independence of properties from the many possible interleavings of the individual actions of a concurrent system. In our experimental context, actions correspond to statements of Promela (the model specification language of SPIN and HSF-SPIN).

To be practically useful, a reduction of the state space must be achieved on-the-fly, during the construction and traversal of the state space. This means that it must be decided per state which transitions, and hence which subsequent states, must be considered. Let $\mathcal{T}=(S, \hat{s}, A, \tau)$ be some LTS.

Definition 2 (Reduction). For any reduction function $r: S \rightarrow 2^{A}$, we define the (partial-order) reduction of $\mathcal{T}$ with respect to $r$ as the smallest LTS $\mathcal{T}_{r}=$ $\left(S_{r}, \hat{s}_{r}, A, \tau_{r}\right)$ satisfying the following conditions:

$-S_{r} \subseteq S, \hat{s}_{r}=\hat{s}$

- for every $s \in S_{r}$ and $a \in r(s)$ such that $\tau(s, a)$ is defined, $\tau_{r}(s, a)=\tau(s, a)$. 
Note that the definition implies that, for every $s \in S_{r}$ and $a \in A$, if $\tau_{r}(s, a)$ is defined, then also $\tau(s, a)$ is defined and $\tau_{r}(s, a)=\tau(s, a)$. Formally, if the function $r(s)$ is fixed in advance, the reduced LTS $\mathcal{T}_{r}$ is independent of the particular algorithm with which it is generated. In practice $r(s)$ is computed onthe-fly during the generation of $\mathcal{T}_{r}$, so the latter may depend on the algorithm.

Not all reductions preserve all properties of interest. Depending on the properties that a reduction must preserve, we have to define additional restrictions on $r$. To this end, we need to formally capture the notion of independence. Actions occurring in different processes can easily influence each other, for example, when they access global variables. The following notion of independence defines the absence of such mutual influence: two independent actions neither disable nor enable one another and they are commutative.

Definition 3 (Independence of actions). Actions $a, b \in A$ with $a \neq b$ are independent in a given state $s \in S$ iff the following holds:

- if $a \in \operatorname{enabled}(s)$ then $b \in \operatorname{enabled}(s)$ iff $b \in \operatorname{enabled}(\tau(s, a))$,

- if $b \in \operatorname{enabled}(s)$ then $a \in \operatorname{enabled}(s)$ iff $a \in \operatorname{enabled}(\tau(s, b))$, and

- $\tau(\tau(s, a), b)=\tau(\tau(s, b), a)$

Actions that are not independent are called dependent. The following conditions are sufficient for preservation of deadlocks [8, 9, 19, 24]:

- C0a: if $a \in r(s)$ then $a \in \operatorname{enabled}(s)$

- Cob: $r(s)=\emptyset$ iff enabled $(s)=\emptyset$.

- C1 (persistence): For any $s \in S$ and execution sequence $s_{0} \stackrel{a_{0}}{\rightarrow} s_{1} \stackrel{a_{1}}{\rightarrow} \ldots \stackrel{a_{n-1}}{\rightarrow}$ $s_{n}$ of length $n \in \mathbb{N} \backslash\{0\}$ such that $s_{0}=s$ and $a_{i} \notin r(s)$ for all $i \in \mathbb{N}$ with $0 \leq i<n$, it holds: action $a_{n-1}$ is independent in $s_{n-1}$ with all actions in $r(s)$.

In this paper we focus on subclasses of safety properties that include Promela assertions [1] (annotations stating the truth of a predicate). (See also the comments in the paragraph after Theorem 1 below.)

The main obstacle in the verification of safety properties is the action ignoring problem which was identified for the first time in 25 . Informally, the ignoring problem occurs when a reduction of a state space ignores the actions of an entire process. For instance, if there is a cyclic process in the system which contains only globally independent actions, i.e., does not interact with the rest of the system, the reduction algorithm could ignore the rest of the system by choosing only actions of this process in $r(s)$. An action $a$ is ignored in a state $s \in S_{r}$ iff $a \in \operatorname{enabled}_{\mathcal{T}}(s)$ and for all $s^{\prime}$ which are reachable in $\mathcal{T}_{r}$ from $s$ it holds $a \notin$ enabled $_{\mathcal{T}_{r}}\left(s^{\prime}\right)$. An action is ignored in $\mathcal{T}_{r}$ iff it is ignored in some state $s \in S_{r}$. So, the following condition prevents action ignoring:

- C2ai: For every $s \in S_{r}$ and every $a \in A$, if $a \in \operatorname{enabled}_{\mathcal{T}}(s)$, then there exists an execution sequence $s_{0} \stackrel{a_{0}}{\rightarrow} s_{1} \stackrel{a_{1}}{\rightarrow} \ldots s_{n-1} \stackrel{a_{n-1}}{\rightarrow} s_{n}$ such that $s=s_{0}$ and which is in the reduced state space $\mathcal{T}_{r}$ (i.e., $s_{i} \in S_{r}$ for $1 \leq i \leq n$ and $a_{i} \in r\left(s_{i}\right)$ for $\left.0 \leq i \leq n-1\right)$ and $a \in r\left(s_{n}\right)$. 
In other words, each delayed transition in $s$ must be eventually executed in a state reachable from $s$.

Condition C2ai implies that each execution sequence (of the original state space) $\sigma$ starting in $s$ has a representative in the reduced state space. A representative violates the safety property iff the sequence in the non-reduced state space violates the property (e.g. [1]). If we see the execution sequence as a sequence of actions, this representative is a permutation of an action sequence obtained by extending $\sigma$ with another (possibly empty) action sequence $\sigma^{\prime}$ from the original state space. More formally, the claim is given by the following theorem:

Theorem 1. Given an LTS $\mathcal{T}$ and a reduction function $r$ that satisfies C0a, C0b, C1, and C2ai, let $s_{0} \stackrel{a_{0}}{\rightarrow} s_{1} \stackrel{a_{1}}{\rightarrow} \ldots s_{n-1} \stackrel{a_{n-1}}{\rightarrow} s_{n}$ be a finite execution sequence of $\mathcal{T}$, such that $s_{0} \in S_{r}$. Then there exists (in $\mathcal{T}$ ) an execution sequence $s_{n} \stackrel{a_{n}}{\rightarrow} s_{1} \stackrel{a_{n+1}}{\rightarrow} \ldots s_{n+k-1} \stackrel{a_{n+k-1}}{\rightarrow} s_{n+k},(k \geq 0)$, such that in $\mathcal{T}_{r}$ there exists an execution sequence $s_{0} \stackrel{a_{\pi(0)}}{\rightarrow} s_{1}^{\prime} \stackrel{a_{\pi(1)}}{\rightarrow} \ldots s_{n+k-1}^{\prime} \stackrel{a_{\pi(n+k-1)}}{\longrightarrow} s_{n+k}$, where $a_{\pi(0)}, a_{\pi(1)}, \ldots, a_{\pi(n+k-1)}$ is a permutation of $a_{0}, a_{1}, \ldots, a_{n+k-1}$.

Proof of the above theorem can be found in 25. Analogous results were proven previously using different versions of the condition that prevents action ignoring (e.g. [8]). Theorem 1 is a meeting point of almost all existing POR-like techniques. It implies preservation of various classes of safety properties (for instance, see 26] for an overview). Among them are also Promela assertions that can be fitted in a straightforward way in one of the existing approaches like assertions in the sense of [8, 12, fact transitions of [25], or local properties of [1].

\subsection{Directed Model Checking}

Explicit-state model checking is primarily state space search. For memory efficiency reasons, the most commonly used algorithms are DFS for safety property verification and nested DFS for liveness property checking. The verification of safety properties can be performed with BFS, which is rather memory inefficient in comparison with DFS. To be able to reconstruct paths to states, BFS needs to store a predecessor link with each state. In addition, the search horizon in BFS grows exponentially with the depth while only linearly in DFS. See [15] for further details. However, BFS guarantees to find an error on an optimally short path. Since short paths into property violating states are helpful in debugging, the authors of [5] suggested the use of heuristically guided search algorithms such as best-first search $(\mathrm{BF})$ and $\mathrm{A}^{*}$ in the state space search, an approach to which they refer to as directed model checking (DMC). Such algorithms hold the potential of locating safety property violating states on short or even optimally short error paths while requiring less states to be stored than BFS. They accomplish this by functions that heuristically assign to each state a value representing the desirability of exploring it. Typical heuristics, for instance, estimate the distance of a state to the set of error states. The heuristic function takes structural properties of the state space as well as properties of the requirements specification into account. 


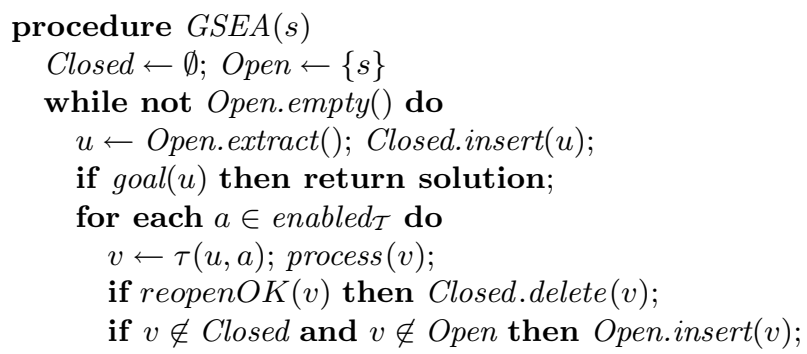

Fig. 1. A general state expanding search algorithm

In this paper we base the construction of a cycle proviso for partial-order reduction on a general search algorithm skeleton that we refer to as general state expanding algorithm (GSEA), c.f. Figure 1] This algorithm divides the set of system states $S$ into three mutually disjoint sets: the set Open of visited but not yet expanded states, the set Closed of visited and expanded states, and the set of unvisited states. The algorithm performs the search by extracting states from Open and moving them into Closed. States extracted from Open are expanded, i.e., the respective successor states are generated. If a successor of an expanded state is neither in Open nor in Closed it is added to Open. Based on the processing done by function reopen $O K$ (line 8) a state can be reopened, i.e., after it is deleted from Closed (line 8) it is reinserted in Open (line 9). DFS (respectively, BFS) can be defined as an instance of the general algorithm presented above, that do not perform reopening of states and where Open is implemented as a stack (resp., queue). Notice that GSEA is not guaranteed to terminate. The termination depends on the state reopening policy, i.e., on the function reopenOK. However, in the sequel we consider only instances for which the termination is guaranteed.

Successful heuristic search algorithms include the non-optimal algorithm BF and the optimal algorithm $A^{*}\left[10\right.$. We present a variant of $A^{*}$ suitable to verify safety properties in Figure 2. It can also be considered a variant of GSEA if one interprets Open as a priority queue in which the priority of a state $v$ is determined by a value $f$. The $f$-value for a state $v$ is computed as the sum of $i$ ) the length $v . g$ of the currently shortest path from the start state to $v$ and $i i$ ) the estimated distance $h(v)$ from $v$ to a goal state. A $^{*}$ can perform a reopening of states. This means that it can move states from Closed to Open when they are reached along a path that is shorter than any path that they were reached on earlier. It is necessary to reopen states in order to guarantee that the algorithm will find the shortest path to the goal state when non-monotone heuristics are used. Monotone heuristics satisfy the property that for each state $u$ and each successor $v$ of $u$ the difference between $h(u)$ and $h(v)$ is less than or equal to the cost of the transition that goes from $u$ to $v$. Note that we usually consider that each transition has a unit cost of 1 , corresponding to the step distance between adjacent states. However, our algorithmic framework can easily handle non unit costs as well. If 
(1) procedure $A^{*}(s)$

(2) begin

(3) Closed $\leftarrow \emptyset ;$ Open $\leftarrow \emptyset ; s . f \leftarrow h(s) ; s . g \leftarrow 0 ;$ Open.insert $(s)$;

(4) while not Open.empty() do

(5) $\quad u \leftarrow$ Open.extractmin(); Closed.insert $(u)$;

(6) if $\operatorname{goal}(u)$ then return solution;

( 7) for each $a \in$ enabled $_{\mathcal{T}}(u)$ do

(8) $\quad v \leftarrow \tau(u, a) ; v . g \leftarrow u . g+\operatorname{cost}(a) ; f^{\prime} \leftarrow v . g+h(v)$;

(9) if $v \in$ Open then

$$
\text { if }\left(f^{\prime}<v . f\right) \text { then } v . f \leftarrow f^{\prime}
$$

else if $v \in$ Closed then

if $\left(f^{\prime}<v . f\right)$ then $v . f \leftarrow f^{\prime} ;$ Closed.delete $(v)$; Open.insert $(v)$;

else $v . f \leftarrow f^{\prime} ;$ Open.insert $(v)$;

Fig. 2. A* search algorithm

non-monotone heuristics are applied, the number of reopenings can be exponential in the size of the state space. However, even if many of the heuristics that we use cannot be proven to be monotone, experimental experience has shown that in practical protocol validation examples states are very rarely reopened [6]. An interesting property of $\mathrm{A}^{*}$ is that if $h$ is a lower bound of the distance to a goal state, then $A^{*}$ will always return the shortest path to a goal state [18].

A key challenge in directed model checking is determining appropriate heuristics. In precursory work, heuristics based on the structure of the property specification, in particular on the syntactic structure of LTL formulae, on local state machine distances as well as property specific heuristics, for instance for deadlock detection, were developed and experimentally evaluated. For more information on directed model checking, as well as the tool HSF-SPIN we refer to the papers [5, 6].

When applying partial-order reduction in the context of directed model checking one is faced with two challenges: a) The pruning of a part of the state space leads to suboptimality of the combined method since optimal error traces may be cut away by the reduction. Experimental results [6] show that in practical examples the sub-optimal solutions are very close to the optimal solutions, if a discrepancy can be detected at all. b) Algorithms such as BF and A* lack a search stack, hence a stack based action prevention condition, such as it is used when implementing partial-order reduction for DFS based state space exploration, cannot be used. The authors of [6] therefore applied two independent over-approximations of the cycle proviso that do not rely on the presence of a search stack, c.f. our discussion in Section 3 .

\section{Action Ignoring Prevention Condition for General Space Exploration}

Condition C2ai from Section 2.2 is stated as a global property of the state space and as such it is expensive to check. Therefore, for practical purposes it 
is important to have a possibly stronger condition (which implies C2ai), but which can be locally checked in an efficient way. For particular state expanding strategies such stronger versions of the ignoring condition exist. For instance for DFS there exists a simple locally checkable condition. For each expanded state $s$ in the reduced state space we require that there exists at least one action $a$ in the reduced action set $r(s)$ and a state $s^{\prime} \in S_{r}$ such that $s \stackrel{a}{\rightarrow} s^{\prime}$ and $s^{\prime}$ is not on the DFS stack. In other words, at least one transition from $r(s)$ must lead to a transition outside the stack, i.e., must not close a cycle. Otherwise, $r(s)=$ enabled $_{\mathcal{T}}(s)$. An analogous version of this condition exists also for BFS [2].

The partial-order reduction version of the general state expanding algorithm (POR GSEA) differs from the original of Figure 1 in line 6 only, where enabled $_{\mathcal{T}}(u)$ is substituted by $r(u)$. We now put the emphasis on the new version of the action ignoring prevention condition.

The conditions that ensure persistence of $r, \mathrm{C} 0 \mathrm{a}, \mathrm{C} 0 \mathrm{~b}$ and $\mathrm{C} 1$, do not depend on the search order, as is argued in [5]. Consequently, they may remain unchanged. Only the condition for ignoring prevention should be adjusted to comply with the general search.

To prevent action ignoring we require that for the currently expanded state $s$ at least one action of $r(s)$ leads to a state $s^{\prime}$ that will be processed later by the algorithm. This means that $s^{\prime}$ is unvisited or it has been visited already but it is in the Open set. The intuition is that the solution to the ignoring problem is postponed until state $s^{\prime}$ is expanded later. The actions which are temporarily ignored in $s$ remain enabled in $s^{\prime}$. This is because by the persistence condition they are independent from the actions in $r(s)$ and therefore they cannot be disabled. Under the assumption that the algorithm terminates, i.e., that the Open set eventually becomes empty, such a postponement will eventually stop. This is because we will eventually arrive at a state for which all transitions lead to states outside Open. For such a state our condition does not hold and therefore the set of explored actions cannot be reduced since at that point we are guaranteed that all possibly postponed actions will be explored.

So, we require that the reduced set (reduction function) $r(u)$, besides conditions $\mathrm{C} 0 \mathrm{a}, \mathrm{C} 0 \mathrm{~b}$ and $\mathrm{C} 1$, has to satisfy for each state $u \in S_{r}$ immediately before its use in the algorithm (the line in POR GSEA corresponding to line 6 of the original algorithm depicted in Figure 1) also the following condition:

- C2c (closed): There exists at least one action $a \in r(u)$ and a state $v \in S_{r}$ such that $u \stackrel{a}{\rightarrow} v$ and $v \notin$ Closed. Otherwise, $r(u)=$ enabled $_{\mathcal{T}}(u)$.

We show below that $\mathrm{C} 2 \mathrm{c}$ implies that the ignoring prevention condition C2ai is satisfied too by the reduced state space, which further entails (via Theorem 1) preservation of safety properties by the POR GSEA algorithm.

Lemma 1. Let $\mathcal{T}=(S, \hat{s}, A, \tau)$ be an LTS with a reduction function $r$ that satisfies conditions COa, C0b, C1, and C2c. Further, let us assume that the POR 
GSEA algorithm terminates when applied on the initial state $\hat{s}$ and produces the reduction $\mathcal{T}_{r}$. Then $r$ satisfies the ignoring prevention condition C2ai.

Proof. The proof is by induction on the (decreasing) order in which the states are removed from Open. As in general each state can be reinserted in Open several times, we establish the ordering based on the last removal of the state. To this end we assign to each state a number $n \in \mathbb{N}$, which we call the removal order of the state. The state which is removed as the very last is assigned the number $\left|S_{r}\right|-1$, where $\left|S_{r}\right|$ is the number of states in $S_{r}$, while the one which is removed first is assigned 0. Such an ordering is always possible because of the assumption that POR GSEA terminates. As a consequence, the set Open eventually becomes empty and there exists some state $s$ which is removed last from the Open set.

Base case: Let $s$ be the state with the highest removal order, i.e., $s$ is removed as the last from Open. Consider the very last removal of $s$ from Open. Since Open is empty, all successors of $s$ must be in Closed. (If they were new they would have been inserted in Open which is a contradiction.) So, by condition C2c, $r(s)=\operatorname{enabled}_{\mathcal{T}}(s)$, i.e., all enabled actions will be explored. The prevention condition C2ai holds trivially.

Inductive step: Let $s$ be the state with removal order $n$. We assume that for each state $s^{\prime \prime}$ with removal order greater than $n$, i.e., which is removed for the last time from Open after $s$ is removed for the last time, the following holds: for each $a \notin r\left(s^{\prime \prime}\right)$, there exists a state $s^{\prime}$ reachable via an execution sequence in the reduced state space such that $a \in r\left(s^{\prime}\right)$. Consider the very last removal of $s$ from Open. If $r(s)=$ enabled $_{\mathcal{T}}(s)$ C2ai holds trivially. So, let us assume that $r(s)$ is a proper subset of enabled $_{\mathcal{T}}(s)$. By condition C2c there exists at least one action $b \in r(s)$ and a state $s^{\prime \prime} \in S_{r}$ such that $s \stackrel{b}{\rightarrow} s^{\prime \prime}$ and $s^{\prime \prime} \notin$ Closed. This implies that $s^{\prime \prime}$ is either a new unvisited state and it will be inserted in Open or it is already in Open. As by our assumption $s$ is already removed (before it is expanded) for the last time from Open (line 4 of the POR GSEA algorithm) we are sure that $s^{\prime \prime}$ will be removed from Open for the last time after $s$. Let $a$ be an action which is not in $r(s)$, i.e., it is postponed. By the persistence condition $\mathrm{C} 1$ actions $a$ and $b$ are independent and therefore $a$ is enabled in $s^{\prime \prime}$. By the induction hypothesis there exists a state $s^{\prime}$ reachable from $s^{\prime \prime}$ via a transition sequence in the reduced state space. The concatenation of $s \stackrel{a}{\rightarrow} s^{\prime \prime}$ and the execution sequence from $s^{\prime \prime}$ to $s^{\prime}$ gives the desired execution sequence from $s$ to $s^{\prime}$.

After proving the termination of the concrete version of the POR GSEA algorithm, its correctness follows by Lemma 1 and further by Theorem 1. Evidently, termination of the concrete version of the POR GSEA algorithm depends on the reopening strategy. Practical strategies, however, guarantee termination. For a deeper discusion, proofs of termination of $A^{*}$ and similar directed search algorithms discussed in Section 2.3 can be found in Section 3.1.2 of [21. As the POR versions of those algorithms work on a subset of the original state space it is trivial to adapt the argument from [21] to the case of the state space reduced by partial-order reduction. Another argument for the termination of the instances of (POR) GSEA is given in [3]. 
In full analogy with the DFS case 22,13, accompanied with some additional restrictions on $r$ [7,23, a stronger version of the open set proviso that preserves $L T L_{-X}$ and $C T L_{-X}^{*}$ (e.g. [4]) can be defined:

- C2cl: (closed liveness) For all actions $a \in r(s)$ and states $s^{\prime} \in S_{r}$ such that $s \stackrel{a}{\rightarrow} s^{\prime}, s^{\prime} \notin$ Closed.

We refer the reader to [3] for further details.

We now turn to the problem of finding efficiently computable cycle provisos for $\mathrm{A}^{*}$. Using the observation made in [14 to prevent global cycles one has to break all local cycles of the involved concurrent processes, in 6 6 a static POR method was adapted to the $\mathrm{A}^{*}$ based directed model checking setting. The method relies on marking one action in every local control cycle as "sticky". It is then enforced that no sticky action is allowed in an ample set of a state if the state is not fully expanded. The resulting proviso $\mathrm{c} 2 \mathrm{~s}$ is defined as the following condition (for the details we refer to the literature) on the reduced set $r(s)$ of a state $s$ state being expanded.

- C2s (static): There exists no sticky action $a \in r(s)$ such that $s \stackrel{a}{\rightarrow} s^{\prime}$. Otherwise, $r(s)=\operatorname{enabled}_{\mathcal{T}}(s)$.

A second idea proposed in [6] was to enforce breaking cycles by requiring that at least one transition in the ample set does not lead to a previously visited state, which lead to the following condition:

- $\mathrm{C} 2 \mathrm{v}$ (visited): There exists at least one action $a \in r(s)$ and a state $s^{\prime} \in S_{r}$ such that $s \stackrel{a}{\rightarrow} s^{\prime}$ and $s^{\prime} \notin$ Closed $\cup$ Open. Otherwise, $r(s)=\operatorname{enabled}_{\mathcal{T}}(s)$.

It is worth noting that our proviso is better than the visited proviso described in the previous section. This is simply because $\mathrm{C} 2 \mathrm{c}$ trivially implies $\mathrm{C} 2 \mathrm{v}$. In the experimental section we will show that, in practice, C2c performs significantly better than $\mathrm{C} 2 \mathrm{v}$.

For safety properties it was shown that $\mathrm{C} 2 \mathrm{~s}$ and $\mathrm{C} 2 \mathrm{v}$ entail the original cycle proviso [6]. Further, while strictly weaker than condition C2ai, experimental results show that still significant reductions could be achieved with these conditions.

\section{Experiments}

This section presents experimental results that evaluate the performance of the proposed proviso. We implemented the approach described in our paper in the tool HSF-SPIN [5] and performed various experiments in which we compare our proposed proviso with the performance of other, previously proposed provisos for BFS and $\mathrm{A}^{*}$. Experiments were performed under Linux on a $\mathrm{PC}$ with an AMD Athlon 1.8 Ghz processor. We use various models in our experiments: A leader election algorithm (leader) that solves the problem of finding a leader in a ring topology, a model of a concurrent program that solves the stable marriage 
problem (marriers $(\mathrm{n}))$, the CORBA GIOP protocol (giop $(\mathrm{n}, \mathrm{m})$ ) which is a key component of the OMG's Common Object Request Broker Architecture (CORBA) specification, and the preliminary design of a Plain Old Telephony System (pots). A description of these models can be found in [5]. Note that these models have been used in benchmarking partial order reductions before, and that the GIOP and POTS models have real-life system complexities. For parameterized scalable models we indicate the instantiated parameters using brackets after the name of the protocol.

Our first set of experiments is devoted to a specific case of the GSEA, namely BFS. None of the previous works on BFS with PO [2,5] presents a comparison with the newly proposed proviso (C2c). The results of [5], which do not consider $\mathrm{C} 2 \mathrm{c}$, show that none between the visited proviso $(\mathrm{C} 2 \mathrm{v})$ [5] and the static proviso (C2s) [14] is better than the other. In contrast, the results of [2] do not consider $\mathrm{C} 2 \mathrm{~s}$ but show that an instance of $\mathrm{C} 2 \mathrm{c}$ for BFS is significantly better than $\mathrm{C} 2 \mathrm{v}$. The main question to investigate is therefore how $\mathrm{C} 2 \mathrm{c}$ performs in comparison to $\mathrm{C} 2 \mathrm{~s}$. Table 1 depicts results obtained by completely exploring the state space of some models using BFS as search algorithm in combination with various reduction methods: no partial-order reduction at all (no), no action ignoring prevention (C2i), C2v, C2s and $\mathrm{C} 2 \mathrm{c}$. Note that $\mathrm{C} 2 \mathrm{i}$ leads to an unsound reduction. We introduce it only in order to assess the other provisos in terms of the number of ample sets that they refuse. For each experiment we present the size of the state space $(\mathrm{s})$, the amount of memory required $(\mathrm{m})$, and the running time $(\mathrm{r})$.

The first thing we observe is that $\mathrm{C} 2 \mathrm{c}$ performs better than $\mathrm{C} 2 \mathrm{v}$. This, for instance, becomes especially obvious in the case of the giop model where $\mathrm{C} 2 \mathrm{c}$ explores about three times less states. Regarding the comparison with the C2s approach, the $\mathrm{C} 2 \mathrm{c}$ based reduction performs better in all cases. Here, the leader model is the most significant example since $\mathrm{C} 2 \mathrm{c}$ explores almost four times less states. Finally, by comparing the colums $\mathrm{C} 2 \mathrm{c}$ and $\mathrm{C} 2 \mathrm{i}$ we observe that $\mathrm{C} 2 \mathrm{c}$

Table 1. Completely exploring state spaces with BFS and several reduction methods

\begin{tabular}{|c|c|c|c|c|c|}
\hline \multicolumn{6}{|c|}{ marriers(3) } \\
\hline & $\mathrm{BFS}+\mathrm{no}$ & $\mathrm{BFS}+\mathrm{C} 2 \mathrm{i}$ & $\mathrm{BFS}+\mathrm{C} 2 \mathrm{v}$ & $\mathrm{BFS}+\mathrm{C} 2 \mathrm{~s}$ & $\mathrm{BFS}+\mathrm{C} 2$ \\
\hline $\mathrm{s}$ & 96,295 & 29,501 & 56,345 & 57,067 & 29,50 \\
\hline $\mathrm{m}$ & 12 & MB & & $8 \mathrm{MB}$ & \\
\hline & $1.13 \mathrm{~s}$ & $0.21 \mathrm{~s}$ & $0.58 \mathrm{~s}$ & & ק \\
\hline \multicolumn{6}{|c|}{ leader $(6)$} \\
\hline & $\mathrm{BFS}+\mathrm{no}$ & $\mathrm{BFS}+\mathrm{C} 2 \mathrm{i}$ & $\mathrm{BFS}+\mathrm{C} 2 \mathrm{v}$ & $\mathrm{BFS}+\mathrm{C} 2 \mathrm{~s}$ & $\mathrm{BFS}+\mathrm{C} 2$ \\
\hline $\mathrm{s}$ & 445,776 & 3,160 & 5,209 & 11,921 & 3,16 \\
\hline $\mathrm{m}$ & $147 \mathrm{MB}$ & $3 \mathrm{MB}$ & $4 \mathrm{MB}$ & $6 \mathrm{MB}$ & $3 \mathrm{M}$ \\
\hline & $34.48 \mathrm{~s}$ & $0.07 \mathrm{~s}$ & $0.18 \mathrm{~s}$ & 0.19 & 0.08 \\
\hline \multicolumn{6}{|c|}{ giop $(2,1)$} \\
\hline & $\mathrm{BFS}+\mathrm{no}$ & $\mathrm{BFS}+\mathrm{C} 2 \mathrm{i}$ & $\mathrm{BFS}+\mathrm{C} 2 \mathrm{v}$ & $\mathrm{BFS}+\mathrm{C} 2 \mathrm{~s}$ & $\mathrm{BFS}+\mathrm{C} 2$ \\
\hline & 664,376 & 65,964 & 209,382 & 231,102 & 66,16 \\
\hline $\mathrm{m}$ & $384 \mathrm{MB}$ & $39 \mathrm{MB}$ & $122 \mathrm{MB}$ & $134 \mathrm{MB}$ & $39 \mathrm{M}$ \\
\hline & $16.42 \mathrm{~s}$ & $1.12 \mathrm{~s}$ & $4.76 \mathrm{~s}$ & $4.44 \mathrm{~s}$ & \\
\hline
\end{tabular}


refuses ample sets in the giop model only. Note that when the exploration with $\mathrm{C} 2 \mathrm{c}$ results in equal state spaces as when ignoring the proviso, there is a small difference in the running time that can be traced to the overhead caused by computing the proviso.

We continue the evaluation of our C2c proviso in a different setting, namely where the goal is error detection and directed model checking algorithms like $\mathrm{A}^{*}$ are used. We also performed additional experiment with other DMC algorithms like best-first search leading to similar results. The results of [5] show no clear winner between $\mathrm{C} 2 \mathrm{v}$ and the $\mathrm{C} 2 \mathrm{~s}$. Hence, the first question to answer is whether $\mathrm{C} 2 \mathrm{c}$ outperforms C2s. Second we would like to find out to what degree $\mathrm{C} 2 \mathrm{c}$ is actually superior to $\mathrm{C} 2 \mathrm{v}$.

To answer this last question we basically extend the results presented in 5 with C2c. Table 2 depicts the results. As in the previous set of experiments, $\mathrm{C} 2 \mathrm{c}$ performs significantly better than $\mathrm{C} 2 \mathrm{v}$. Consider, for instance, the models marriers and giop, where the number of states explored with $\mathrm{C} 2 \mathrm{c}$ is only about half the number explored with $\mathrm{C} 2 \mathrm{v}$. On the other hand, there is no clear winner between $\mathrm{C} 2 \mathrm{c}$ and $\mathrm{C} 2 \mathrm{~s}$ approach. For instance, the best reduction is achieved with $\mathrm{C} 2 \mathrm{~s}$ in model marriers and with $\mathrm{C} 2 \mathrm{c}$ in model giop. In the rest of the models both provisos work equally well.

By comparing the two previous sets of experiments we observe the following phenomenon: in the marriers model, algorithm BFS with C2c explores as many states as BFS with C2i (Table 1), while $\mathrm{A}^{*}$ with $\mathrm{C} 2 \mathrm{c}$ explores almost twice as many states as $\mathrm{A}^{*}$ with $\mathrm{C} 2 \mathrm{i}$ (Table 2). In other words, the $\mathrm{C} 2 \mathrm{c}$ proviso is refuting

Table 2. Finding a safety violation with $\mathrm{A}^{*}$ and BFS with several reduction methods

marriers $(4)$

\begin{tabular}{|c|c|c|c|c|c|c|}
\hline & $\mathrm{A}^{*}+\mathrm{no}$ & $\mathrm{A}^{*}+\mathrm{C} 2 \mathrm{i}$ & $\mathrm{A}^{*}+\mathrm{C} 2 \mathrm{v}$ & $\mathrm{A}^{*}+\mathrm{C} 2 \mathrm{~s}$ & $\mathrm{~A}^{*}+\mathrm{C} 2 \mathrm{c}$ & $\mathrm{BFS}+\mathrm{C} 2 \mathrm{c}$ \\
\hline $\mathrm{s}$ & 225,404 & 37,220 & 100,278 & 37,220 & 58,500 & 155,894 \\
\hline $\mathrm{m}$ & $31 \mathrm{MB}$ & $7 \mathrm{MB}$ & $15 \mathrm{MB}$ & $7 \mathrm{MB}$ & $6 \mathrm{MB}$ & $22 \mathrm{MB}$ \\
\hline $\mathrm{r}$ & $5.15 \mathrm{~s}$ & $0.31 \mathrm{~s}$ & $2.99 \mathrm{~s}$ & $0.36 \mathrm{~s}$ & $0.73 \mathrm{~s}$ & $7.17 \mathrm{~s}$ \\
\hline \multicolumn{7}{|c|}{ pots } \\
\hline & $\mathrm{A}^{*}+\mathrm{no}$ & $\mathrm{A}^{*}+\mathrm{C} 2 \mathrm{i}$ & $\mathrm{A}^{*}+\mathrm{C} 2 \mathrm{v}$ & $\mathrm{A}^{*}+\mathrm{C} 2 \mathrm{~s}$ & $\mathrm{~A}^{*}+\mathrm{C} 2 \mathrm{c}$ & $\mathrm{BFS}+\mathrm{C} 2 \mathrm{c}$ \\
\hline $\mathrm{s}$ & 6,654 & 5,429 & 5,574 & 5,429 & 5,429 & 22,786 \\
\hline $\mathrm{m}$ & $5 \mathrm{MB}$ & $4 \mathrm{MB}$ & $4 \mathrm{MB}$ & $4 \mathrm{MB}$ & $4 \mathrm{MB}$ & $12 \mathrm{MB}$ \\
\hline $\mathrm{r}$ & $0.18 \mathrm{~s}$ & $0.15 \mathrm{~s}$ & $0.15 \mathrm{~s}$ & $0.15 \mathrm{~s}$ & $0.15 \mathrm{~s}$ & $0.78 \mathrm{~s}$ \\
\hline \multicolumn{7}{|c|}{ leader $(8)$} \\
\hline & $\mathrm{A}^{*}+\mathrm{no}$ & $\mathrm{A}^{*}+\mathrm{C} 2 \mathrm{i}$ & $\mathrm{A}^{*}+\mathrm{C} 2 \mathrm{v}$ & $\mathrm{A}^{*}+\mathrm{C} 2 \mathrm{~s}$ & $\mathrm{~A}^{*}+\mathrm{C} 2 \mathrm{c}$ & $\mathrm{BFS}+\mathrm{C} 2 \mathrm{c}$ \\
\hline $\mathrm{s}$ & 558,214 & 104 & 104 & 104 & 104 & 128 \\
\hline $\mathrm{m}$ & $265 \mathrm{MB}$ & $2 \mathrm{MB}$ & $2 \mathrm{MB}$ & $2 \mathrm{MB}$ & $2 \mathrm{MB}$ & $2 \mathrm{MB}$ \\
\hline $\mathrm{r}$ & $30.54 \mathrm{~s}$ & $0.01 \mathrm{~s}$ & $0.01 \mathrm{~s}$ & $0.01 \mathrm{~s}$ & $0.01 \mathrm{~s}$ & $0.01 \mathrm{~s}$ \\
\hline
\end{tabular}

giop $(3,1)$

\begin{tabular}{|l|r|r|r|r|r|r|}
\hline & $\mathrm{A}^{*}+\mathrm{no}$ & $\mathrm{A}^{*}+\mathrm{C} 2 \mathrm{i}$ & $\mathrm{A}^{*}+\mathrm{C} 2 \mathrm{v}$ & $\mathrm{A}^{*}+\mathrm{C} 2 \mathrm{~s}$ & $\mathrm{~A}^{*}+\mathrm{C} 2 \mathrm{c}$ & $\mathrm{BFS}+\mathrm{C} 2 \mathrm{c}$ \\
\hline $\mathrm{s}$ & 485,907 & 90,412 & 314,964 & 191,805 & 117,846 & 120,132 \\
$\mathrm{~m}$ & $291 \mathrm{MB}$ & $55 \mathrm{MB}$ & $189 \mathrm{MB}$ & $116 \mathrm{MB}$ & $72 \mathrm{MB}$ & $73 \mathrm{MB}$ \\
$\mathrm{r}$ & $20.09 \mathrm{~s}$ & $2.82 \mathrm{~s}$ & $12.41 \mathrm{~s}$ & $6.60 \mathrm{~s}$ & $3.98 \mathrm{~s}$ & 2,52 \\
\hline
\end{tabular}


ample sets when the search algorithm is $\mathrm{A}^{*}$ but not when it is BFS. What happens is that the new proviso, as well as the rest of the provisos, depends on the order in which states are explored. This phenomenon can be illustrated by a simple example. Assume the following state space:

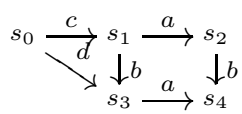

Suppose that $\hat{s}=s_{0}$ and that actions $a, b$ are unconditionally independent and that we use BFS with our proviso to explore the state space. First, state $s_{0}$ is extracted from the open set and its successors $s_{1}, s_{3}$ are inserted into Open (we assume that no reduction is possible at $s_{0}$ ). Assume that the order in which they are inserted is $s_{1}$ first and then $s_{3}$. At the next iteration of BFS, state $s_{1}$ is selected for expansion. Now, $\{b\}$ is selected as ample set since it satisfies all the conditions. In the last step state $s_{4}$ is explored. The algorithm, hence, explores all states but $s_{2}$.

Consider now that $s_{3}$ is inserted in Open first and $s_{1}$ second. Now, state $s_{3}$ is extracted from the Open set and $s_{4}$ is inserted in it. In the next step, state $s_{2}$ is selected for expansion, but this time set $\{b\}$ is refused by C2c since state $s_{3}$ is no more in the open set. Thus, the search is forced to visit state $s_{4}$. In sum, the whole state space is visited.

We have performed some experiments in which the exhaustive exploration is performed randomly. This was done by using the $\mathrm{A}^{*}$ algorithm and a random heuristic function. The result leads to larger state spaces than with BFS. At this point an interesting question arises. While previous work presents the benefits of using directed search algorithms over BFS, can BFS when used with C2c take advantage of the exploration order phenomenon so as to become more memory efficient than $\mathrm{A}^{*}$ with $\mathrm{C} 2 \mathrm{c}$ ? This is particularly relevant since partial-order reduction holds the potential of containing the state space explosion that BFS is particularly vulnerable to. To answer this question we included experiments with BFS and $\mathrm{C} 2 \mathrm{c}$ in Table 2. With the $\mathrm{C} 2 \mathrm{c}$ proviso $\mathrm{A}^{*}$ explores less states than BFS with $\mathrm{C} 2 \mathrm{c}$. While in the pots and marriers models the improvement is significant, in giop the small difference together with the overhead introduced by heuristics leads to slightly longer running times for $\mathrm{A}^{*}$.

\section{Conclusions}

In this paper we presented a partial-order reduction for general state exploring algorithms. The main novelty in the algorithm lies in the condition for avoiding action ignoring, which we call open set proviso, which is basically a generalization of the queue proviso proposed for SPIN's BFS based partial-order reduction in 2. During the state space exploration this condition can be checked locally and in an efficient way. We implemented the open set proviso for some directed model checking algorithms which are special instances of the general search algorithm. The experimental results show that the new proviso leads to a significant 
performance improvement of the directed model checking algorithms in comparison to previously known provisos. The experiments also showed that $\mathrm{A}^{*}$ together with the open list proviso is performing superior in terms of explored states and memory consuption over BFS with partial-order reduction and this new proviso.

We notice that the efficiency of the proviso can depend on the order in which the actions in the reduced state set are selected. In addition, further experiments we have performed evidence that when there are various valid ample sets the choice amongst them influcences size of the reduced state space. It could be interesting to see if this can be exploited to further improve POR algorithms. In particular, we propose to investigate whether heuristics, possibly exploiting the property being verified, can be defined to select amongst different prossibleample sets in order to improve efficiency of the reduction. Another interesting topic for future work will be to apply the ideas of this paper in the realm of symbolic model checking, for instance, for the verification of liveness properties.

\section{References}

1. R. Alur, R.K. Brayton, T.A. Henzinger, S. Qadeer, and S.K. Rajamani, Partialorder reduction in symbolic state-space exploration, Formal Methods in System Design, 18:97-116, 2001. A preliminary version appeared in Proc. of the 9th International Conference on Computer-aided Verification, CAV '97, LNCS 1254, pp. 340-351, Springer, 1997.

2. D. Bošnački, G.J. Holzmann, Improving Spin's Partial-Order Reduction for Breadth-First Search, Model Checking Software: 12th International SPIN Workshop, SPIN 2005, LNCS 3639, pp.91-105, Springer, 2005.

3. D. Bošnački, S. Leue, A. Lluch Lafuente, Partial-Order Reduction for General State Exploring Algorithms, Technical Report soft-05-02, Chair for Software Engineering, University of Konstanz, 2005.

http://www.inf.uni-konstanz.de/soft/research/publications/pdf/soft-05-01.pdf

4. E. Clarke, O. Grumberg, D.A. Peled, Model Checking MIT Press, 2000.

5. S. Edelkamp, A. Lluch Lafuente and S. Leue, Directed explicit-state model checking in the validation of communication protocols, Software Tools for Technology Transfer, vol. 5, pp. 247-267, 2004.

6. S. Edelkamp, S. Leue and A. Lluch Lafuente, Partial-order reduction and trail improvement in directed model checking, International Journal on Software Tools for Technology Transfer, vol. 6, nr. 4, pp. 277-301, 2004.

7. R. Gerth, R. Kuiper, D. Peled, W. Penczek, A Partial-Order Approach to Branching Time Logic Model Checking, Information and Computation 150(2): 132-152, 1999.

8. P. Godefroid, Partial-Order Methods for the Verification of Concurrent Systems: An Approach to the State Space Explosion, LNCS 1032, Springer, 1996.

9. P. Godefroid, P. Wolper, Using Partial-Orders for the Efficient Verification of Deadlock Freedom and Safety Properties, Computer Added Verification, CAV '91, LNCS 575, pp. 332-342, Springer, 1991.

10. P.E. Hart, N.J. Nilsson and B. Raphael, A formal basis for heuristic determination of minimum path costs, IEEE Transactions on Systems Science and Cybernetics, 4:100-107, 1968. 
11. G.J. Holzmann, The SPIN Model Checker: Primer and Reference Manual, Addison Wesley, 2003.

12. G.J. Holzmann, P. Godefroid, D. Pirottin, Coverage Preserving Reduction Strategies for Reachability Analysis, in Proc. 12th IFIP WG 6.1. International Symposium on Protocol Specification, Testing, and Validation, FORTE/PSTV '92, pp.349-363, North-Holland, 1992.

13. G.J. Holzmann, D. Peled, An Improvement in Formal Verification, FORTE 1994, Bern, Switzerland, 1994.

14. R.P. Kurshan, V. Levin, M. Minea, D. Peled, H. Yenigün, Static Partial-Order Reduction, in Tools and Algorithms for Construction and Analysis of Systems TACAS '98, LNCS 1384, pp. 345-357, 1998.

15. A. Lluch-Lafuente, S. Edelkamp, S. Leue, Directed Search for the Verification of Communication Protocols, PhD Thesis, Freiburger Dokument Server, Institute of Computer Science, University of Freiburg, June 2003.

16. V. Levin, R. Palmer, S. Qadeer, S.K. Rajamani, Sound Transaction-Based Reduction Without Cycle Detection, Model Checking Software: 12th International SPIN Workshop, SPIN 2005, LNCS 3639, pp.106-121, Springer, 2005.

17. R. Nalumasu, G. Gopalakrishnan, An Efficient Partial-Order Reduction Algorithm with an Alternative Proviso Implementation, Formal Methods in System Design 20(3): 231-247, 2002.

18. N.J. Nilsson, Principles of Artificial Intelligence, Tioga Publishing Co. Palo Alto, California, 1980.

19. W.T. Overman, Verification of Concurrent Systems: Function and Timing, Ph.D. Thesis, UCLA, Los Angeles, California, 1981.

20. R. Palmer, G. Gopalakrishnan, A Distributed Partial Order Reduction Algorithm, Formal Techniques for Networked and Distributed Systems FORTE 2002, LNCS 2529, p.370, 2002.

21. J. Pearl, Heuristics, Addison-Wesley, 1985

22. D.A. Peled, Combining Partial-Order Reductions with On-the-Fly Model Checking, Formal Methods on Systems Design, 8: 39-64, 1996. A previous version appeared in Computer Aided Verification 1994, LCNS 818, pp. 377-390, 1994.

23. B. Willems, P. Wolper, Partial-Order Models for Model Checking: From Linear to Branching Time, Proc. of 11 Symposium of Logics in Computer Science, LICS 96, New Brunswick, pp. 294-303, 1996.

24. A. Valmari, Eliminating Redundant Interleavings during Concurrent Program Verification, Proc. of Parallel Architectures and Languages Europe '89, vol. 2, LNCS 366, pp. 89-103, Springer, 1989.

25. A. Valmari, A Stubborn Attack on State Explosion, in Advances in Petri Nets, LNCS 531, pp. 156-165, Springer, 1991.

26. A. Valmari, The State Explosion Problem, Lectures on Petri Nets I: Basic Models, LNCS Tutorials, LNCS 1491, pp. 429-528, Springer, 1998. 